\title{
Effectiveness and Tolerability of Micafungin in Chinese Patients with Invasive Fungal Infections: A Retrospective, Multicenter Study
}

\author{
Xiaoyun Zheng · Xiaobo Huang · Jianmin Luo · Juan Li • \\ Wei Li · Qifa Liu · Ting Niu · Xiaodong Wang · Jianfeng Zhou • \\ Xi Zhang · Jianda $\mathrm{Hu} \cdot$ Kaiyan Liu
}

Received: March 13, 2018 / Published online: August 13, 2018

(C) The Author(s) 2018

\section{ABSTRACT}

Introduction: Invasive fungal infections (IFIs) are a significant health problem in immunocompromised patients, resulting in substantial morbidity, mortality, and healthcare costs. Micafungin is a broad-spectrum echinocandin

Enhanced digital features To view enhanced digital features for this article go to https://doi.org/10.6084/ m9.figshare.6865253.

Electronic supplementary material The online version of this article (https://doi.org/10.1007/s12325018-0762-5) contains supplementary material, which is available to authorized users.

\section{Zheng}

Fujian Institute of Hematology, Fujian Provincial

Key Laboratory on Hematology, Fujian Medical

University Union Hospital, Fujian, China

X. Huang $\cdot$ X. Wang

Sichuan Provincial Peoples' Hospital, Sichuan,

China

J. Luo

The Second Hospital of Hebei Medical University, Hebei, China

J. Li

The First Affiliated Hospital, Sun Yat-sen University,

Guangdong, China

W. Li

The First Hospital of Jilin University, Jilin, China

Q. Liu

Nanfang Hospital, Guangdong, China with activity against Candida and Aspergillus spp. This was a multicenter, non-comparative, retrospective observational study that evaluated the effectiveness and tolerability of intravenously administered micafungin for treating IFIs caused by Candida and Aspergillus spp.

Methods: Adult patients in China who had received at least one dose of intravenously administered micafungin were eligible. Retrospective data (May 2008-April 2015) were extracted from patients' medical files and recorded using electronic data capture. The primary endpoint was overall success rate (patients with complete or partial response). Subgroup analyses determined effectiveness

T. Niu

West China Hospital of Sichuan University, Sichuan, China

J. Zhou

Tongji Medical College Huazhong University of Science and Technology, Hubei, China

X. Zhang

XinQiao Hospital, Third Military Medical

University, Chongqing, China

J. Hu (凶)

Fujian Institute of Hematology, Fujian Provincial Key Laboratory on Hematology, Fujian Medical University Union Hospital, Fujian, China e-mail: drjiandahu@163.com

K. Liu (ه)

Peking University Institute of Hematology, Peking University People's Hospital, Beijing, China e-mail: liukaiyan@medmail.com.cn 
according to diagnostic certainty, fungal species, type of IFI, duration of micafungin treatment, and daily dose of micafungin. Tolerability, including the incidence of adverse events (AEs), was also assessed.

Results: Overall, 2555 patients who received at least one dose of micafungin were identified. The mean duration of treatment and mean daily dose were 10.2 days and $133.0 \mathrm{mg}$, respectively. The overall success rate was $60.8 \%$; this was significantly higher in patients who received treatment for at least 1 week (range $67.9-71.6 \%$ [mean $69.2 \%])$ compared with less than 1 week $(47.8 \%$; $P<0.0001)$, and those who received 50-100 mg (65.7\%) compared with other daily doses (range 42.9-60.1\% [mean 59.0\%]; $P=0.0011$ ). Success rates in Candida- and Aspergillus-infected patients were similar $(61.9 \%$ and $56.8 \%$, respectively). AEs and adverse drug reactions were observed in $36.2 \%$ and $4.5 \%$ of patients, respectively. The majority of AEs were mild, while discontinuation due to AEs was low (2.3\%).

Conclusion: Micafungin is effective and well tolerated for the treatment of patients with IFIs in China, as demonstrated in Candida- and Aspergillus-infected adults. Subgroup analyses highlighted the potential benefits of treating IFIs with micafungin for a minimum of 1 week. Trial registration: ClinicalTrials.gov identifier NCT02678598.

Funding: Astellas Pharma Inc.

Keywords: Aspergillus; Candida; Invasive fungal infections; Micafungin; Real-world data; Retrospective study

\section{INTRODUCTION}

Invasive fungal infections (IFIs) represent an important clinical challenge in immunosuppressed patients, causing substantial morbidity and mortality that result in increased healthcare costs $[1,2]$. IFIs are opportunistic and their occurrence has risen in recent decades because of increased numbers of patients undergoing procedures such as hematopoietic stem cell and solid organ transplantation, and receiving chemotherapies for autoimmune conditions and malignancies $[1,3,4]$. In addition, the relative contribution of individual species to IFIs has changed markedly over the last three decades [4]. Candida and Aspergillus are the most commonly reported pathogenic yeast and mold infections in immunocompromised patients in China [5]. The incidence of these infections is currently increasing, which has been attributed to higher rates of hospitalization due to an aging population and an increase in the occurrence of chronic diseases [5].

Micafungin is an echinocandin with broadspectrum activity against Candida and Aspergillus, as shown in vitro using clinical isolates of these genera [6, 7]. Numerous studies have demonstrated the efficacy and safety of this agent for the treatment of invasive Candida and Aspergillus infections [1, 8], although licensing of micafungin to treat these infections varies between countries.

In China, micafungin has demonstrated efficacy in the prevention of IFIs in neutropenic patients undergoing hematopoietic stem cell transplant. In an open-label study comparing the efficacy and safety of micafungin vs. itraconazole in this population, overall success rates, as measured by the absence of an IFI, were $92.6 \%$ and $94.6 \%$, respectively, and better treatment tolerance was observed with micafungin [9]. In a separate study of renal transplant patients with IFIs in China, similar efficacy rates (74.2\% vs. $78.8 \%)$ were observed between micafungin and voriconazole, respectively [10]. Mortality rates in the micafungin and voriconazole groups were $9.7 \%$ and $12.1 \%$, respectively. Both drugs were generally well tolerated with $41.9 \%$ and $51.6 \%$ of patients experiencing adverse events (AEs) in the micafungin and voriconazole groups, respectively [10].

The aim of the current retrospective study was to evaluate the real-world effectiveness and tolerability of intravenously administered micafungin for IFIs caused by Candida or Aspergillus spp. in adult patients in China.

\section{METHODS}

\section{Study Design and Treatment}

This multicenter, non-comparative, retrospective observational study (ACN-MA-2014-02; 
Clinicaltrials.gov ID NCT02678598) was conducted at 35 sites in 26 hospitals in China (sites could include hematology departments and intensive care units within the same hospital). Micafungin was administered according to clinicians' usual clinical practice, and data on all outcome measures were extracted from patients' medical files and recorded using electronic data capture. In China, the recommended dose range of micafungin is $50-150 \mathrm{mg}$, which can be increased up to $300 \mathrm{mg}$ /day for severe or refractory candidiasis or aspergillosis; the recommended duration of treatment is 2 and 6-12 weeks in Candida- and Aspergillus-infected patients, respectively, with treatment dose and duration determined according to the severity of disease and the patient's condition. The observation period was the time between the first dose of micafungin and the end/discontinuation of treatment.

\section{Patients}

The study evaluated patients who had received at least one dose of intravenously administered micafungin between May 2008 and April 2015. Male or female patients, aged 18 years or more who were prescribed micafungin as empirical (driven by a pre-emptive diagnosis according to European Organization for Research and Treatment of Cancer/Invasive Fungal Infections Cooperative Group and the National Institute of Allergy and Infectious Diseases Mycoses Study Group [EORTC/MSG] criteria) [11] or targeted antifungal therapy were considered for participation. Patients were excluded if they received concomitant administration of other antifungal agents, and if the patient's medical records were missing data and/or unclear.

All procedures performed in studies involving human participants were in accordance with the ethical standards of the institutional and/or national research committee and with the 1964 Helsinki declaration and its later amendments or comparable ethical standards. Informed consent was obtained from all individual participants included in the study.

\section{Disease Characteristics}

Assessments performed included the overall diagnoses (i.e., possible, probable, or proven), the type of IFI (i.e., Aspergillus or Candida, if confirmed), any identifiable disease, the duration of treatment, assessment of fever, imaging examinations (reported as normal or abnormal findings), and mycological examinations (e.g., including blood or sputum culture, $\beta$-D-glucan test, and microscopy of sputum culture; reported as positive or negative). Resolution of fever, other symptoms and signs, imaging abnormalities, and mycological evidence of pathogen clearance were subsequently evaluated at the end/discontinuation of treatment and used to inform assessments for the primary endpoint. All assessments and diagnoses were performed according to the judgment of the treating physician, who worked within the diagnostic framework of EORTC/MSG criteria.

\section{Outcome Measures}

\section{Effectiveness}

The primary endpoint was the overall success rate, which was defined as the proportion of patients with complete or partial responses. The proportions of patients who died, had disease progression, or had a stable response were also calculated. Definitions of the response categories used are summarized in Supplementary Table 1.

\section{Subgroup Analyses for Effectiveness}

Subgroup analyses were conducted to evaluate the effectiveness of micafungin according to the diagnostic certainty (possible, probable, and proven); fungal organism (Candida or Aspergillus); the type of invasive disease (fungemia, respiratory mycosis [suspected or clinically confirmed pneumonia due to Candida or Aspergillus spp.], or gastrointestinal mycosis [suspected or confirmed gastroenteritis, i.e., diarrhea and abdominal pain, or incidence confirmed by fecal culture, respectively]); the duration of micafungin treatment $(<1, \geq 1$ to $<2$ weeks, $\geq 2$ to $<4$ weeks, and $\geq 4$ weeks); and the daily dose of micafungin (0-50, 50-100, 100-150, 150-200, and 200-300 mg). 


\section{Tolerability}

The secondary endpoints were the incidences of AEs and adverse drug reactions (ADRs). ADRs were defined as events that were, in the opinion of the investigator, possibly or probably related to micafungin treatment. Serious ADRs that occurred from the day of the first dose to 1 week after the end of treatment were included in the analysis. Additional tolerability measures included routine blood, urine, and hepatic and renal function tests conducted during treatment. Any clinically relevant changes in these parameters were recorded as AEs or ADRs.

\section{Statistical Analyses}

Enrollment of 3000 patients across 35 sites was planned. For the effectiveness analyses, the primary population was the full analysis set (FAS), defined as all patients whose records showed that they had received at least one dose of micafungin and had an effectiveness assessment. Effectiveness analyses were also conducted using a per-protocol set (PPS) which comprised all subjects who received at least 1 week of treatment (for Aspergillus infection with hematology disease, this was at least 4 weeks) and who did not meet any of the following criteria: inclusion/exclusion violations; micafungin treatment combined with acyclovir or ganciclovir that affected the assessment of study endpoints (based on reduced potency of micafungin when received in combination with these products, as mentioned in the Chinese package insert for micafungin); dosage of micafungin that exceeded $300 \mathrm{mg}$ /day; or offlabel use of micafungin. The tolerability analyses were conducted in a safety analysis set (SAS), which was defined as all patients who received at least one dose of micafungin and had at least one safety assessment.

All data were reported descriptively, using means accompanied by asymptotic $95 \%$ confidence intervals (CI) to indicate precision. The effect of treatment duration and dose on the overall treatment success rates were analyzed using a chi-square test; $P$ values less than 0.05 were required for the difference between two groups to be considered significant. Analyses were performed using Statistical Analysis System version 9.3 , or above.

\section{RESULTS}

\section{Patients}

The study was conducted between January and December 2015, with April 30, 2015 as the end time point for patients receiving micafungin treatment. Overall, data from 2611 patients were screened, and 56 excluded, resulting in 2555 enrolled patients whose records showed that they had received at least one dose of micafungin (Fig. 1). These patients were included in the SAS, while the FAS and PPS comprised 2467 and 1457 patients, respectively. No patients were excluded from the PPS as a result of acyclovir or ganciclovir affecting the assessment of study endpoints. The mean (standard deviation; SD) age was $56( \pm 18.9)$ years and most patients $(61.4 \%)$ were male. Disease characteristics (including the confirmed diagnosis, the organism causing the infection, the type of disease, and the duration of treatment) are summarized in Table 1 . The majority of patients had a possible diagnosis $(n=1717$ [69.6\%]), while the remaining patients had probable $(n=699$ [28.3\%]) or proven $(n=51$ [2.1\%]) diagnoses. Most patients did not undergo imaging examinations, but abnormalities were reported in up to $37.1 \%$ who received any single type of imaging examination. Overall, 1192 $(48.3 \%)$ patients received a mycological examination; $17.1 \%$ of patients in the FAS had a positive result. The infecting organism was not established in 2092 (84.8\%) cases; however, where the organism was identified, a higher proportion were infected with Candida (294 [11.9\%]) than Aspergillus (81 [3.3\%]). Respiratory mycosis was the most frequently encountered fungal disease $(n=1063 ; 43.1 \%)$; the infected site was not established in over half of cases $(n=1325 ; 53.7 \%)$. Diagnoses with fever before receiving micafungin was reported in 1403 [57.1\% of 2458]) patients assessed. Corresponding data for the PPS population (organism causing the infection, type of disease, and 


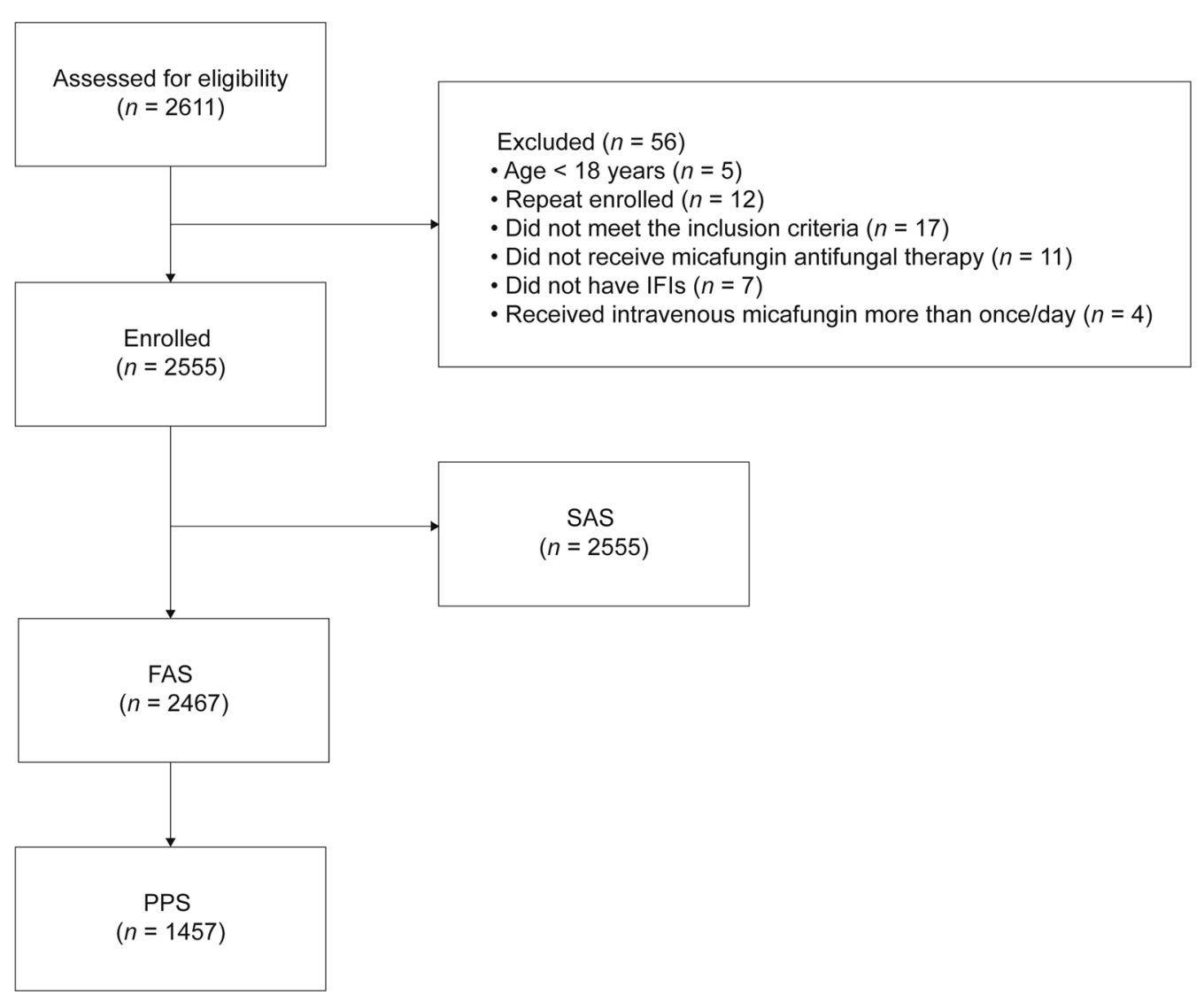

Fig. 1 Patient disposition. FAS full analysis set, IFI invasive fungal infection, PPS per-protocol set, $S A S$ safety analysis set

duration of treatment) are summarized in Supplementary Table 2 .

\section{Treatment}

The mean duration of micafungin treatment was 10.2 days (95\% CI 9.9-10.6) and the mean daily dose was $133.0 \mathrm{mg}$ (95\% CI 131.6-134.3).

\section{Outcome Measures}

\section{Effectiveness}

The overall success rate in the FAS (primary endpoint) was $60.8 \% \quad(95 \%$ CI $58.8-62.7)$ (Table 2). Rates of complete response, partial response, stable disease, disease progression, and mortality in the FAS are also summarized in Table 2. Complete responses were observed in $17.2 \%$ (95\% CI $15.7-18.7)$ of patients and partial responses in $43.5 \%$ (95\% CI 41.6-45.5). The overall mortality rate was $6.7 \% \quad(95 \%$ CI 5.7-7.7). Rates of resolution of fever, other signs and symptoms, imaging abnormalities, and mycological evidence of pathogen clearance in the FAS are summarized in Supplementary Table 3.

In the PPS, the overall success rate was $69.1 \%$ (95\% CI 66.7-71.5). Results for rates of complete response, partial response, stable disease, disease progression, and mortality were similar to those in the FAS (Supplementary Table 4).

\section{Subgroup Analyses for Effectiveness}

The overall success rates according to the subgroup analyses in the FAS are shown in Fig. 2. Overall success rates were numerically higher among patients with a possible or a probable diagnosis (61.1\% [95\% CI 58.8-63.4] and 60.7\% [95\% CI 57.0-64.3], respectively), compared with those who had a proven diagnosis $(51.0 \%$ [95\% CI 37.3-64.7]). According to the causative 
Table 1 Distribution of patients according to disease characteristics and the duration of treatment (FAS)

\begin{tabular}{|c|c|}
\hline Diagnostic certainty, $n$ (\%) & $n=2467$ \\
\hline Possible & $1717(69.6)$ \\
\hline Probable & $699(28.3)$ \\
\hline Proven & $51(2.1)$ \\
\hline Type of IFI, $n(\%)$ & $n=2467$ \\
\hline Aspergillus-infected patients & $81(3.3)$ \\
\hline Candida-infected patients & $294(11.9)$ \\
\hline Not identifiable & $2092(84.8)$ \\
\hline Diseases, $n(\%)$ & $n=2467$ \\
\hline Gastrointestinal mycosis ${ }^{\mathrm{a}}$ & $23(0.9)$ \\
\hline Fungemia & $56(2.3)$ \\
\hline Respiratory mycosis ${ }^{\mathrm{b}}$ & $1063(43.1)$ \\
\hline Not identifiable & $1325(53.7)$ \\
\hline Duration of treatment, $n$ (\%) (weeks) & $n=2467$ \\
\hline$\geq 4$ & $102(4.1)$ \\
\hline$\geq 2$ to $<4$ & $518(21.0)$ \\
\hline$\geq 1$ to $<2$ & $874(35.4)$ \\
\hline$<1$ & $973(39.4)$ \\
\hline Fever, $n(\%)$ & $(n=2458)^{\mathrm{c}}$ \\
\hline Yes & $1403(57.1)^{\mathrm{d}}$ \\
\hline No & $986(40.1)$ \\
\hline Not examined & $69(2.8)$ \\
\hline \multicolumn{2}{|l|}{ Imaging examinations, $n(\%)^{\mathrm{e}}$} \\
\hline Chest radiography & $n=2467$ \\
\hline Normal & $23(0.93)^{\mathrm{f}}$ \\
\hline Abnormal & $438(17.8)^{\mathrm{f}}$ \\
\hline Not examined & $2006(81.3)$ \\
\hline Chest CT & $(n=2462)^{g}$ \\
\hline Normal & $20(0.8)^{\mathrm{h}}$ \\
\hline Abnormal & $914(37.1)^{\mathrm{h}}$ \\
\hline Not examined & $1528(62.1)$ \\
\hline Other imaging examination & $(n=2465)^{\mathrm{i}}$ \\
\hline Normal & $15(0.6)^{j}$ \\
\hline
\end{tabular}

Table 1 continued

\begin{tabular}{ll}
\hline Abnormal & $92(3.7)^{\mathrm{j}}$ \\
Not examined & $2358(95.7)$ \\
Mycological examinations, $n(\%)$ & $n=2467$ \\
Positive & $422(17.1)$ \\
Negative & $770(31.2)$ \\
Not examined & $1275(51.7)$ \\
\hline
\end{tabular}

$C T$ computed tomography, FAS full analysis set, IFI invasive fungal infection, $S D$ standard deviation

${ }^{a}$ Gastrointestinal mycoses were suspected or confirmed gastroenteritis (diarrhea and abdominal pain, or incidence confirmed by fecal culture, respectively)

${ }^{\mathrm{b}}$ Respiratory mycoses included suspected or clinically confirmed pneumonia due to Candida or Aspergillus spp.

${ }^{c}$ Data unavailable from nine patients; ${ }^{\mathrm{g}}$ five patients; and ${ }^{i}$ seven patients for fever, chest CT, and other imaging examinations, respectively

${ }^{d}$ Body temperature (mean $[ \pm S D]$ ) of patients with fever was $38.6{ }^{\circ} \mathrm{C}( \pm 0.7)$; data were collected from 1400 patients (missing data, $n=3$ )

${ }^{\mathrm{e}}$ Patients may have received more than one type of imaging examination

${ }^{\mathrm{f}}$ The longest diameter of the lesion was measurable for $n=6\left(0.2 \%\right.$ of 438 patients); ${ }^{\mathrm{h}} n=42$ (1.7\% of 914 patients); and ${ }^{j} n=5$ (0.2\% of 92 patients) for chest radiography, chest $\mathrm{CT}$, and other imaging examinations, respectively

organism, overall success rates were similar for Candida-infected patients (61.9\%) vs. Aspergillus-infected patients (56.8\%). Stratification according to disease type suggested higher resolution among patients with respiratory mycosis $(62.9 \%)$ compared with fungemia $(55.4 \%)$ or gastrointestinal mycosis (43.5\%). From a statistical perspective, the overall success rate was significantly higher in each group of patients who received treatment for a longer duration; i.e., $\geq 1$ week (range 67.9-71.6\%; mean 69.2\%), compared with $<1$ week $(47.8 \%)$ (chi-square test $P<0.0001$ ), including the comparison of $\geq 1$ to $<2$ weeks' treatment duration compared with $<1$ week $(47.8 \%$ vs. $67.9 \%$, respectively; $P<0.0001)$. When stratification was performed by daily dose, the highest overall success rates were reported with doses of 50-100 mg (65.7\%) 
Table 2 Response, progression, and mortality rates during micafungin treatment (FAS $[n=2467]$ )

Comprehensive assessment of effectiveness, $n$ (\%); 95\% $\mathrm{CI}^{\mathrm{a}}$

Complete response

$$
\begin{aligned}
& 425 \text { (17.2); } 15.7-18.7 \\
& 1074 \text { (43.5); } 41.6-45.5 \\
& 473 \text { (19.2); } 17.6-20.7 \\
& 330 \text { (13.4); } 12.0-14.7 \\
& 165 \text { (6.7); 5.7-7.7 }
\end{aligned}
$$

Partial response

Stable response

Progression of disease

Death

Evaluation of overall success rate of patients at the end of treatment, $n$ (\%), 95\% CI

Failure

$$
968 \text { (39.2); } 37.3-41.2
$$

Response

1499 (60.8); 58.8-62.7

$C I$ confidence interval, $F A S$ full analysis set

${ }^{a}$ Asymptotic 95\% CIs of the percentage response rate calculated

Fig. 2 Overall success rates (proportion of patients with complete or partial response) at the end of treatment according to diagnostic certainty (a); the organism causing the infection (b); type of disease (c); treatment duration (d); and the daily dose (e) in the FAS. $P$ values indicate significant differences between treatment groups (providing there is also a clear difference in the proportion of patients with a complete or partial response); error bars represent asymptotic $95 \%$ confidence intervals. $F A S$ full analysis set

and 100-150 mg (60.1\%); significant differences were reported for these doses compared with a higher daily dose (e.g., 200-300 mg [42.9\%]) $(P=0.0011)$.

Corresponding results for patients in the PPS are shown in Supplementary Fig. 1. Overall success rates in subgroups defined by the diagnostic certainty, fungal organism, and disease type were slightly higher than those in the FAS, which reflects exclusion from the PPS of patients who received treatment for less than 1 week. Consistent with the FAS, significant differences were reported when stratification was performed by daily dose $(P=0.0003)$;
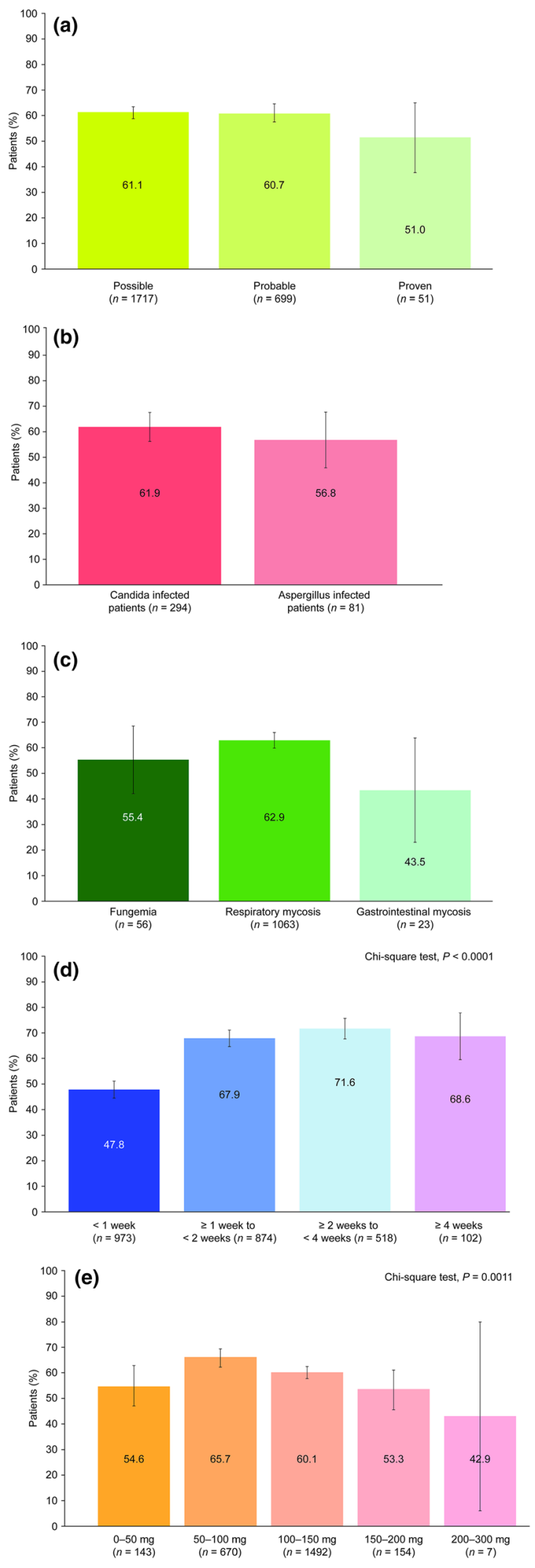
Table 3 Overall tolerability profile of micafungin (SAS $[n=2555])$

\begin{tabular}{lcc}
\hline & $\begin{array}{l}\text { Number of } \\
\text { patients (\%) }\end{array}$ & $\begin{array}{l}\text { Number of } \\
\text { events }\end{array}$ \\
\hline Overall AEs & $925(36.2)$ & 1989 \\
Mild & $606(23.7)$ & 1150 \\
Moderate & $266(10.4)$ & 414 \\
Severe & $269(10.5)$ & 425 \\
ADRs considered to be & $116(4.5)$ & 177 \\
$\quad$ drug related & $4(0.2)$ & 6 \\
Probably related & $113(4.4)$ & 171 \\
Possibly related & $190(7.4)$ & 297 \\
SAEs & $5(0.2)$ & 9 \\
Serious ADRs & $59(2.3)$ & 92 \\
AEs leading to & & \\
discontinuation & & \\
\hline
\end{tabular}

$A D R s$ adverse drug reactions, AEs adverse events, SAEs serious AEs, $S A S$ safety analysis set

however, there was no significant effect of treatment duration on overall success rates in the PPS.

\section{Tolerability}

The overall tolerability profile of micafungin is summarized in Table 3 . In total, AEs were observed in 925 (36.2\%) patients and ADRs were observed in $116(4.5 \%)$ patients. In most cases (more than 50\%), the severity of the AEs was mild. AEs that occurred in more than $1 \%$ of patients are shown in Supplementary Table 5; the most common AEs were dyspnea, diarrhea, and pulmonary infection, observed in 58 $(2.3 \%), 56(2.2 \%)$, and $55(2.2 \%)$ patients, respectively. The most common ADRs were abnormal hepatic function (19 patients [0.7\%]); diarrhea (14 patients [0.6\%]); rash (12 patients [0.5\%]); and decreased platelet counts, fever, and peripheral edema (5 patients each [0.2\%]).

Fifty-nine patients $(2.3 \%)$ discontinued as a result of AEs. Five patients $(0.2 \%)$ had nine serious ADRs, which were possibly related to the study drug, and one of these patients discontinued treatment. The ADRs comprised one patient who experienced pulmonary infection, disseminated intravascular coagulation, and multiple organ failure; one patient who experienced postoperative sepsis with liver metastasis of gastric cancer; one patient who experienced shortness of breath and chest tightness; one patient who experienced respiratory failure; and one patient who experienced septic shock. Two patients experienced severe ADRs that were lifethreatening. Three patients $(0.1 \%)$ with severe ADRs died: one patient who experienced pulmonary infection, disseminated intravascular coagulation, and multiple organ failure; one who experienced respiratory failure; and one who experienced postoperative sepsis and liver metastasis associated with gastric cancer.

\section{DISCUSSION}

This retrospective analysis in adult patients in China provides real-world evidence that intravenously administered micafungin is effective against IFIs caused by Aspergillus and Candida when used as empirical antifungal therapy, driven by a pre-emptive diagnosis and targeted antifungal therapy.

The overall success rates observed in this retrospective analysis were broadly comparable with those seen with other antifungal agents in Chinese patients with IFIs. In a retrospective analysis of data from patients treated with amphotericin B for IFIs, an overall response rate of $76 \%$ was observed [12], while an overall response rate of $67.3 \%$ was observed in a Chinese study evaluating the efficacy of amphotericin B for IFIs in patients with hematologic diseases [13]. In a further randomized study comparing prophylactic posaconazole and fluconazole for the prevention of IFIs in patients with acute myelogenous leukemia or myelodysplastic syndrome, the overall incidences of possible, probable, or proven IFIs were $9.4 \%$ and $22.2 \%$ in each treatment group, respectively [14]. Again, it is not possible to draw conclusions regarding the relative efficacy of micafungin vs. these agents as these are not head-to-head evaluations, and such between- 
study comparisons should be made with caution.

Stratification of the findings by treatment duration and the daily dose received indicated that micafungin treatment is most effective when continued for longer than 1 week, or if prescribed between 50 and $100 \mathrm{mg} /$ day. Results suggesting that increasing the treatment length beyond 1 week is more effective than a shorter treatment duration were also reflected in the difference between the overall success rates in the FAS and the PPS, which excluded patients who received treatment for less than 1 week. This is a potentially important finding which emphasizes the importance of treating high-risk patients with IFIs for a minimum duration of 14 days, as demonstrated in recent studies of micafungin in transplant patients in China $[10,15]$, and in accordance with the Chinese summary of product characteristics for micafungin. The results from this study were not stratified by disease severity, but it is possible this may have had an impact on the findings observed for treatment duration.

Findings that suggest micafungin is most effective when dosed between 50 and $100 \mathrm{mg} /$ day are in line with the Chinese label for micafungin (50 or $100 \mathrm{mg}$ for the treatment of patients with invasive candidiasis or aspergillosis), and European and US guidelines for the treatment of invasive candidiasis (which recommend micafungin $100 \mathrm{mg}$ as initial, targeted treatment; the latter in non-neutropenic patients) $[16,17]$. Of note, micafungin is not currently indicated for the treatment of infections caused by Aspergillosis spp. in Europe or the USA $[18,19]$. However, some limitations are clear with regards to the subgroup analyses that were performed. Firstly, there were substantial differences between the patient numbers included in different groups analyzed, with small numbers included in some groups; unfortunately, it was not possible to combine groups after the analyses were performed. Secondly, no objective measure was used to determine a clear difference in percentage overall success rate, which was also required to infer significance if a $P$ value of less than 0.05 was reported; any measures of significance were subjective and are therefore limited. These issues with the subgroup analyses limit the conclusions that can be inferred.

The findings from the current analyses showed that micafungin is well tolerated, with a low incidence of ADRs, serious ADRs, and AEs leading to discontinuation of treatment. Overall, the data add to the growing body of evidence demonstrating the effectiveness and tolerability of micafungin for the treatment of patients with invasive fungal infections; however, it is difficult to compare this trial directly with others because of differences in the patient populations and in the way that treatment success was evaluated [1, 8-10, 20, 21].

The strengths of this study are that it included a large patient population and that it provides real-world evidence of micafungin effectiveness and tolerability in current clinical practice. It should also be noted that the results may represent a conservative estimate of the effectiveness of micafungin, as patients whose symptom resolution data were unavailable were assumed to be non-responders. Limitations of the study include its retrospective design and the lack of a comparator arm; also, the study design did not stratify patients by treatment approach (i.e., empirical- or diagnostic-driven antifungal therapy); therefore, the overall success rates reported were derived using resolution/improvement in fever symptoms alone, in a subset of patients whose data were not further evaluated. The small proportion of patients in the FAS who received imaging or mycological examinations may have contributed to the lack of identifiable disease or type of IFI, and imaging and mycological assessments were limited by the use of inconsistent methodology before and after treatment, or a lack of reassessment following treatment. Also, the small patient numbers included in some subgroups may have limited the statistical analyses. It would therefore be worthwhile conducting a randomized, active-controlled study with micafungin in Chinese patients with IFIs, to confirm the findings reported and provide head-to-head data. Long-term safety data for patients treated with micafungin in a real-world setting would also be beneficial; this is an objective of a large, multicenter, observational cohort study conducted in the USA (MYCOS; ClinicalTrials.gov 
NCT01686607), which is due for completion in January 2020 [22].

\section{CONCLUSION}

This retrospective study in real-world clinical practice indicates that micafungin is effective and well tolerated as antifungal treatment in Chinese adults with IFIs. Furthermore, micafungin appeared to be effective in treating both Candida and Aspergillus infections, irrespective of the diseases evaluated. Notably, worse outcomes were observed in patients with a short duration of treatment; thus, this should be addressed by highlighting a minimum duration for the treatment of IFIs with micafungin, which could be implemented in clinical practice and medical education programs.

\section{ACKNOWLEDGEMENTS}

We thank the participants of the study.

Funding. This study was initiated and funded by Astellas Pharma Inc. The article processing charges and the open access fee were also funded by Astellas Pharma Inc. All authors had full access to all of the data in this study and take complete responsibility for the integrity of the data and accuracy of the data analysis.

Medical Writing Assistance. Medical writing support, which was funded by Astellas Pharma Inc, was provided by Nicola French, PhD and Lucy Kanan, PhD of Bioscript Medical.

Authorship. All named authors meet the International Committee of Medical Journal Editors (ICMJE) criteria for authorship for this article, take responsibility for the integrity of the work as a whole, and have given their approval for this version to be published.

Disclosures. Xiaoyun Zheng, Xiaobo Huang, Jianmin Luo, Juan Li, Wei Li, Qifa Liu, Ting Niu, Xiaodong Wang, Jianfeng Zhou, Xi Zhang,
Jianda $\mathrm{Hu}$, and Kaiyan Liu have nothing to disclose.

Compliance with Ethics Guidelines. All procedures performed in studies involving human participants were in accordance with the ethical standards of the institutional and/or national research committee and with the 1964 Helsinki declaration and its later amendments or comparable ethical standards. Informed consent was obtained from all individual participants included in the study.

Data Availability. The datasets generated during and/or analyzed during the current study are not publicly available due to the Astellas China data policy, but are available from the corresponding author on reasonable request. All data requests will be assessed individually for confidentiality, patient consent, and compliance with applicable regulations prior to being made available.

Open Access. This article is distributed under the terms of the Creative Commons Attribution-NonCommercial 4.0 International License (http://creativecommons.org/licenses/ by-nc/4.0/), which permits any noncommercial use, distribution, and reproduction in any medium, provided you give appropriate credit to the original author(s) and the source, provide a link to the Creative Commons license, and indicate if changes were made.

\section{REFERENCES}

1. de la Torre P, Reboli AC. Micafungin: an evidencebased review of its place in therapy. Core Evid. 2014;9:27-39.

2. Drgona L, Khachatryan A, Stephens J, et al. Clinical and economic burden of invasive fungal diseases in Europe: focus on pre-emptive and empirical treatment of Aspergillus and Candida species. Eur J Clin Microbiol Infect Dis. 2014;33:7-21.

3. Cornely OA. Aspergillus to Zygomycetes: causes, risk factors, prevention, and treatment of invasive fungal infections. Infection. 2008;36:296-313. 
4. Kriengkauykiat J, Ito JI, Dadwal SS. Epidemiology and treatment approaches in management of invasive fungal infections. Clin Epidemiol. 2011;3:175-91.

5. Liao Y, Chen M, Hartmann T, Yang RY, Liao WQ. Epidemiology of opportunistic invasive fungal infections in China: review of literature. Chin Med J (Engl). 2013;126:361-8.

6. Messer SA, Diekema DJ, Boyken L, Tendolkar S, Hollis RJ, Pfaller MA. Activities of micafungin against 315 invasive clinical isolates of fluconazoleresistant Candida spp. J Clin Microbiol. 2006;44:324-6.

7. Pfaller MA, Boyken L, Hollis RJ, et al. In vitro susceptibility of clinical isolates of Aspergillus spp. to anidulafungin, caspofungin, and micafungin: a head-to-head comparison using the CLSI M38-A2 broth microdilution method. J Clin Microbiol. 2009;47:3323-5.

8. Hanadate T, Wakasugi M, Sogabe K, et al. Evaluation of the safety and efficacy of micafungin in Japanese patients with deep mycosis: a post-marketing survey report. J Infect Chemother. 2011;17:622-32.

9. Huang X, Chen H, Han M, et al. Multicenter, randomized, open-label study comparing the efficacy and safety of micafungin versus itraconazole for prophylaxis of invasive fungal infections in patients undergoing hematopoietic stem cell transplant. Biol Blood Marrow Transpl. 2012;18:1509-16.

10. Shang W, Feng G, Sun R, et al. Comparison of micafungin and voriconazole in the treatment of invasive fungal infections in kidney transplant recipients. J Clin Pharm Ther. 2012;37:652-6.

11. De Pauw B, Walsh TJ, Donnelly JP, et al. Revised definitions of invasive fungal disease from the European Organization for Research and Treatment of Cancer/Invasive Fungal Infections Cooperative Group and the National Institute of Allergy and Infectious Diseases Mycoses Study Group (EORTC/ MSG) Consensus Group. Clin Infect Dis. 2008;46:1813-21.

12. Ma XJ, Li GP, Zhou J, Wang A, Li TS. A retrospective study of amphotericin B treatment for invasive fungal infection. Zhonghua Nei Ke Za Zhi. 2007;46:718-20.
13. Jia L, Huang M, Liu WL, et al. Clinical analysis of amphotericin $\mathrm{B}$ in the treatment of invasive fungal infections in 121 patients with hematologic diseases. Zhonghua Xue Ye Xue $\mathrm{Za}$ Zhi. 2008;29:619-22.

14. Shen Y, Huang XJ, Wang JX, et al. Posaconazole vs. fluconazole as invasive fungal infection prophylaxis in China: a multicenter, randomized, open-label study. Int J Clin Pharmacol Ther. 2013;51:738-45.

15. Yan T, Li SL, Wang DX. Comparison between micafungin and caspofungin for the empirical treatment of severe intra-abdominal infections in surgical intensive care patients. Zhonghua Yi Xue Za Zhi. 2016;96:2301-6.

16. Cornely OA, Bassetti M, Calandra T, et al. ESCMID guideline for the diagnosis and management of Candida diseases 2012: non-neutropenic adult patients. Clin Microbiol Infect. 2012;18:19-37.

17. Pappas PG, Kauffman CA, Andes DR, et al. Clinical practice guideline for the management of candidiasis: 2016 update by the Infectious Diseases Society of America. Clin Infect Dis. 2016;62:409-17.

18. Electronic Medicines Compendium. EU summary of product characteristics for micafungin. 2018. https://www.medicines.org.uk/emc/medicine/2099 $7 / \mathrm{SPC} /$ Mycamine $+50 \mathrm{mg}+$ and $+100 \mathrm{mg}+$ powder + for+solution+for+infusion/. Accessed June 2018.

19. Food and Drug Administration. Prescribing information for micafungin. 2018. https://www.astellas. us/docs/mycamine.pdf/. Accessed June 2018.

20. Chen GL, Chen Y, Zhu CQ, Yang CD, Ye S. Invasive fungal infection in Chinese patients with systemic lupus erythematosus. Clin Rheumatol. 2012;31:1087-91.

21. Enoch DA, Idris SF, Aliyu SH, Micallef C, Sule O, Karas JA. Micafungin for the treatment of invasive aspergillosis. J Infect. 2014;68:507-26.

22. Clinicaltrials.gov. Trial record for NCT01686607: Short and long-term safety of micafungin and other parenteral antifungal agents (MYCOS). 2018. https://www.clinicaltrials.gov/ct2/show/NCT01686 607?term=MYCOS\&rank=1/. Accessed 26 April 2018. 